\title{
Adaptive-AR Model with Drivers' Prediction for Traffic Simulation
}

\author{
Xuequan Lu, ${ }^{1}$ Mingliang Xu, ${ }^{2}$ Wenzhi Chen, ${ }^{1}$ Zonghui Wang, ${ }^{1}$ and Abdennour El Rhalibi ${ }^{3}$ \\ ${ }^{1}$ Zhejiang University, Hangzhou 310027, Zhejiang, China \\ ${ }^{2}$ Zhengzhou University, Hangzhou 310027, Zhejiang, China \\ ${ }^{3}$ Liverpool John Moores University, UK \\ Correspondence should be addressed to Wenzhi Chen; chenwz@zju.edu.cn
}

Received 23 June 2013; Revised 9 August 2013; Accepted 3 September 2013

Academic Editor: Ali Arya

Copyright (C) 2013 Xuequan Lu et al. This is an open access article distributed under the Creative Commons Attribution License, which permits unrestricted use, distribution, and reproduction in any medium, provided the original work is properly cited.

We present a novel model called A ${ }^{2}$ R_ “Adaptive-AR"-based on a well-known continuum-based model called AR Aw and Rascle (2000) for the simulation of vehicle traffic flows. However, in the standard continuum-based model, vehicles usually follow the flows passively, without taking into account drivers' behavior and effectiveness. In order to simulate real-life traffic flows, we extend the model with a few factors, which include the effectiveness of drivers' prediction, drivers' reaction time, and drivers' types. We demonstrate that our $\mathrm{A}^{2} \mathrm{R}$ model is effective and the results of the experiments agree well with experience in real world. It has been shown that such a model makes vehicle flows perform more realistically and is closer to the real-life traffic than AR (short for Aw and Rascle and introduced in Aw and Rascle (2000)) model while having a similar performance.

\section{Introduction}

With the world's rapid technological and economic developments in transport, there are an arising number of vehicles on the roads in cities, towns, and countryside all over the world, resulting in a large amount of challenges related to traffic. Accordingly, road traffic research including the modeling, simulation, and visualization of vehicle flows has become paramount for a large number of researchers. Traffic simulation plays an essential role in virtual worlds, especially in sport or simulation games from the entertainment industry. A well-known example of such games is "Need for Speed". Vehicular games typically utilize agent-based traffic models, which involves a significantly growing processing cost when the number of vehicles becomes larger [1]. Therefore, trying to simulate traffic flows by means of macroscopic traffic models, such as $A^{2} R$, is an effective way in vehicular games since macroscopic continuum models are fast and can handle large areas in a virtual world efficiently [1]. In addition, vehicle flows make a big difference in urban development and in the design of roads, as well as improving policies and guidelines with respect to traffic regulation. Furthermore, by exploring vehicle flows, we can investigate the causes of traffic accidents and congestions and study traffic signs impact on road circulation and so on.

As a matter of fact, most vehicle flows are simulated with agent-based microscopic models [1]. This type of model is very popular; however, it requires a great deal of time and energy and needs a lot of computation [1]. As the number of vehicles grows, the total simulation time increases dramatically [1], thus leading to a decrease in overall performance. Another common simulation method is based on continuum-based macroscopic models [1]. This model can optimize the total simulation time to a great extent; however, it just follows the vehicle flows passively, without considering drivers' behavior and effectiveness. To be closer to real-life traffic flow, it is necessary to consider drivers' conduct.

In consideration of the problems mentioned above, a model called " $\mathrm{A}{ }^{2} \mathrm{R}$ " is proposed in this paper. This new model is created on the basis of a continuum-based macroscopic model called AR [2]. We expect our approach not only uses much less simulation time than microscopic methods in large-scale, real-world networks of traffic, but also takes the effectiveness of drivers' prediction into consideration. 
To represent the movement of vehicle flows, we use a computational grid, in which vehicles have their own information enabling a discrete visual representation. As for the drivers' effectiveness in our model, we define it practically as follows: when the flux at a grid point (i.e., interface of two nearest grids along each lane) in the front is larger than that at the current location, the driver at the current location will make an adaptive reaction of accelerating after some time latency and reciprocally. Specifically, drivers' visual ability needs to be considered, which means some grid points in the front should all be taken into account in drivers' decision making. As an illustration, a driver will make some adaptive reaction like accelerating when he or she observes the fluxes of a few grid points clearly; the reaction is supported by the effects of taking into account those grid points. We will describe this in Section 4.

The rest of the paper is structured as follows. We review the related literature in Section 2. In Section 3, we introduce some definitions and formulation of our problem for convenience and briefness. Section 4 introduces our model in detail. More specifically, in Section 4.1, we present an overview of our model. In Section 4.2, we introduce the effectiveness of drivers' prediction, with different driver types, and reaction time. In Section 4.3, our integrated model is reported. In Section 5, we report our experiments with $A^{2} R$, discuss the outcomes, and compare them with AR model. Conclusion and future work are discussed in Section 6.

\section{Related Work}

Many problems and solutions have been studied in traffic simulation; most methods aim at studying and exploring specific phenomena, for example, traffic congestions and stop-and-go patterns.

Most existing methods used in traffic simulation have been classified into three categories: microscopic simulation, macroscopic simulation, and mesoscopic simulation. Among these three classes, microscopic simulation, which is agentbased and in which each vehicle is regarded as an individual, is the most popular one.

Agent-based methods usually establish some complex rules to generate some natural behavior for cars. Gerlough [3], Newell [4], and Algers et al. [5] discussed the carfollowing set of rules. Helbing [6] presented a complete description of differing traffic models. Nagel and Schreckenberg [7] applied cellular automata into the field of traffic dynamics. Treiber et al. [8] discussed traffic states in empirical observations and microscopic simulations. MITSIM [9] and SUMO [10] are agent-based simulation systems.

Macroscopic methods, which are based on continuous models, are common ways to simulate vehicle flows. Lighthill and Whitham [11] and Richards [12] did the earliest work in this area, and formed a model named LWR (i.e., LighthillWhitham-Richards traffic flow model). Combined with the LWR model, Payne [13] proposed a new model called PW by creating a second-order system of equations from gas dynamics, which can depict the nonequilibrium state of car flows. Nevertheless, the PW model might figure out negative velocities under certain conditions. Eventually, Aw and Rascle [2] and Zhang [14] amended the PW model and removed the nonphysical behavior. Our approach is based on the strength of Aw's AR model to simulate car flows.

The third class of traffic simulation is called mesoscopic, using Boltzmann-like mesoscale equations and a continuumbased description. Prigogine and Andrews [15] were the first to come up with this idea. Afterwards, Nelson et al. [16] and Shvetsov and Helbing [17] did a few corresponding improvements from the seminal work.

Nagatani [18] proposed simplified versions of continuum models of traffic to describe the jamming transition in traffic flow, where drivers' sensitivity was taken into account. However, the sensitivity is inversely proportional to delay time, which is quite different from the sensitivity coefficient defined in our model. Based on Nagatani's model, Sun and Tian [19] made improvements by considering the effectiveness of drivers' prediction and proved that it was efficient in enhancing the stability of traffic flow, however they considered only the flux at the grid point ahead from the current position.

There has not been any extensive research, which covered the topics on simulation of vehicles and traffic flows. Go et al. [20] presented a way of animating vehicles in a particular situation, and Sewall et al. [21] took advantage of discrete temporal spatial data to reconstruct traffic flows. Wilkie et al. [22] proposed a self-aware method for traffic route planning in large-scale traffic simulation, and they [23] later put forward an efficient automatic method for extrapolating a road map from a GIS database to automatically create a geometrically correct and topologically consistent 3D model of large-scale road network to be readily used in a real-time traffic simulation, interactive visualization of virtual world, and autonomous vehicle navigation. Recently, Lu et al. [24] animated rural traffic scenes using their proposed accidentavoidance full velocity difference model.

Recently, Sewall et al. [1] have put forward a novel method for the animation and synthesis of realistic traffic flows on large-scale road networks, and it is capable of handling lane changes and merges. Sewall et al. [25] also presented a novel, real-time algorithm for modeling large-scale realistic traffic using a hybrid model of both continuum- and agent-based methods for traffic simulation. However, car flows in their work [1] follow the rules of conservation laws passively. Nonetheless, the evolution of vehicle flows in practice has a close connection with drivers' control, with which car flows may become "active". In order to approximate the realworld traffic flows better, we propose a new approach with the consideration of drivers' predictive efficiency, which not only reflects drivers' subjectivity but also makes car flows "alive".

Briefly, compared with microscopic models, the $\mathrm{A}^{2} \mathrm{R}$ model that we built upon the basis of AR model needs much less computation and simulation time; meanwhile, we bring in drivers' prediction, driver types, and reaction time [26], which can generate adaptive driving behavior, for instance, accelerating and decelerating. Furthermore, we believe that the car flows simulated using our model are much closer to real-life traffic flows than AR model. 


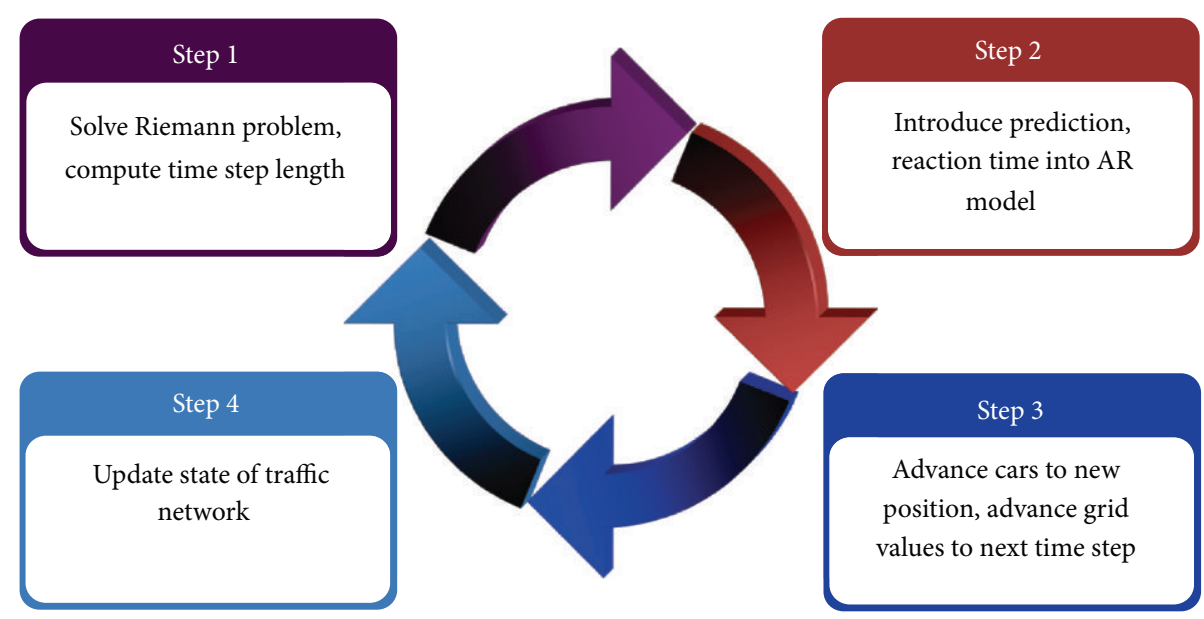

FiguRE 1: The overall process of our model.

\section{Problem Formulation}

To describe our methodology concisely and conveniently, we introduce the following definitions.

Definition 1. The road network is a set of roads, $\mathrm{RN}=\bigcup\left\{R_{z} \mid\right.$ $z=1,2 \ldots\}$.

Definition 2. Each road consists of a number of lanes, and the properties of lane $k$ are $P_{k}=\left\{u_{\max }\right.$, lanes, roads $\}$, where $u_{\max }$ is the maximum speed, lanes are those lanes which are adjacent to lane $k$, and roads are ones which lane $k$ belongs to.

Definition 3. Lane $k$ is divided into discrete grids; the grid length along lane $k$ is $\Delta x_{k}$, and each grid length is the same. $\Delta x$ is a "target" length that all grids should have, and it is greater than the longest vehicle in the simulation. In this paper, we choose $\Delta x$ twice or three times the smallest vehicle size. The number of grids and the grid length are calculated as follows:

$$
N_{k}=\left\lfloor\frac{L_{k}}{\Delta x}\right\rfloor, \quad \Delta x_{k}=\frac{L_{k}}{N_{k}} .
$$

Definition 4. Each lane $k$ contains some cars, $\operatorname{cars}_{k}=\bigcup\left\{c_{i} \mid\right.$ $i=1,2,3 \ldots\}$; each car has a state information, including position, drivers' prediction, and vehicle type. However, if there is no prediction, we do not need to consider reaction time, sensitivity coefficient, and observed distance. $c_{i}=$ \{pos, type, prediction, reaction, sensitivity, observe\}.

Definition 5. The vehicle flows move with continuous time steps $\Delta t$, and $\lambda_{\max k}$ is the maximum speed of waves in lane $k$, then $\Delta t$ should satisfy the Courant-Friedrichs-Lewy (CFL) condition [27], which is a necessary condition of convergence, to make the integration stable. Consider

$$
\Delta t<\min \left(\frac{\Delta x_{k}}{\lambda_{\max k}}\right) .
$$

Definition 6. Based on our experience and common sense, drivers may be generally classified into three categories: some are aggressive, some are conservative, and the others are normal ones. Because of these categories, drivers may have different reaction time. We also incorporate these concepts into our model.

The following equations represent the system of $A R$ model [2]:

$$
\begin{aligned}
& \partial_{t} \rho+\partial_{x}(\rho v)=0, \\
& \partial_{t} y+\partial_{x}(y v)=0,
\end{aligned}
$$

where $\rho$ is the density of traffic, and $y$ is the relative flow of traffic. They both are conservative variables, subscripts $t$ and $x$ denote differentiation in time and space, respectively. In this paper, we use the form of $y$ for continuum traffic simulation based on the model described in [1].

\section{Our $A^{2}$ R Model}

In this section, we describe our method for the simulation of vehicle flows in great detail.

4.1. Overview. The following diagram illustrates the overall process of our $\mathrm{A}^{2} \mathrm{R}$ model.

Combined with Figure 1, given a time step, we take the following steps to simulate the vehicle flows.

Initialization: First of all, we divide each lane into diverse numbers of grids, with the same length of grids in a lane, and specify initial and boundary conditions before starting the simulation.

Step 1. According to the values in grids, we compute the characteristic speeds and fluxes at each interface (i.e., grid point) between grids by solving the Riemann Problem [1] at that interface. Find the largest speed from the computed values above, and compute time step length by satisfying the CFL condition [27]. 
Step 2. Introduce drivers' prediction and reaction time into AR model, and amend the variable $y$ with these factors.

Step 3. Given the values in grids, compute velocity fields, and look where a car lies in. Subsequently, use the explicit 4th-order Runge-Kutta method to integrate each car's new position, and move the cars to the updated place. Advance to the next time step using the numerical discretization form of AR model for each grid, and compute grid values for the next time step.

Step 4. Update the state of the whole traffic network.

Go to Step 1, to run another cycle. It should be noted that time step length computation cannot be skipped, because it ensures the integration stability by fulfilling CFL condition [27].

4.2. Drivers. The variable $y$ in (4) [2] has the following form:

$$
y=\rho v+\rho p(\rho),
$$

where $p$ is an increasing function of traffic density $\rho$. In the system of AR model, $\rho$ should be conservative at all times, but $y$ needs some relaxation.

To represent the subjectivity of car flows, we bring in the behavior and nature of drivers, while driving on roads. As a result, this idea makes the original vehicle flows more "active" and "alive", which approximates the real-life traffic flows.

We describe drivers' prediction, driver types, and reaction time in more details in the following.

4.2.1. Drivers' Prediction. To express drivers' subjectivity, we introduce drivers' prediction which results in accelerating, decelerating, or some other reactions depending on road conditions represented by flux variation at the front grid point. However, we do not modify (4) in the general form, instead we just amend $y$ (i.e., the relative flow of traffic) during the update procedure. The amended formula is as follows:

$$
y(t+\tau)=y(t)+k \frac{\partial \mathbf{Q}(x+\delta)}{\partial x},
$$

where $k$ is the sensitivity coefficient, which reflects the sensitive degree to flux variation at the front grid point. If $k$ is zero, it means that drivers' prediction is not considered and degenerates to the AR model. $\mathbf{Q}(x+\delta)_{x}=\partial \mathbf{Q}(x+\delta) / \partial x$ is the prediction item of flux at front grid point, which denotes drivers' effectiveness to flux variation at front grid point; subscript $x$ denotes differentiation in space. $\tau$ denotes drivers' reaction time, and $\delta$ is the grid length in consistent lanes, so we obtain $\delta=\Delta x_{k}$. That is to say, at time $t$, a driver observes the flux variation at front grid point and makes his or her prediction, after a response time of $\tau$ seconds, this driver will do some action such as acceleration.

We can obtain the following form from (6) using spatial discretization:

$$
y(t+\tau)=y(t)+k \frac{\mathbf{Q}(x+\delta, t)-\mathbf{Q}(x, t)}{\delta} .
$$

From the above equations, we can claim that there is only one grid point in front being considered. But such a restriction cannot describe the drivers' visual ability completely, since a real-life driver makes a prediction on the basis of his or her visibility instead of only the closest grid point to the current position. As a result, we extend the proto model to a more comprehensive model in the last part of Section 4 .

4.2.2. Driver Types. As it is well-known, drivers in real world can be classified into three classes [28]: conservative, aggressive, and normal ones. What is worth mentioning is that the same driver may probably have varying sensitivity coefficients while making decisions for accelerating or decelerating.

Rationally, when the coefficient $k_{+}$under accelerating is notably greater than the coefficient $k_{-}$under decelerating, these drivers are aggressive ones, conversely, they are conservative drivers. If $k_{+}$and $k_{-}$are nearly the same for a single driver, he or she is a normal one. For this reason, we can introduce the following conditions:

Aggressive drivers: $k_{+} \gg k_{-}$, more sensitive when $\Delta Q \geq 0$.

Conservative drivers: $k_{+} \ll k_{-}$, more sensitive when $\Delta Q<0$.

Normal drivers: $k_{+} \approx k_{-}$, almost the same sensitivity whatever and whenever it is.

4.2.3. Reaction Time. As known to all, human beings need some time to make a reaction or response to some stimuli. A large number of experiments have been conducted by neurological researchers and others to prove this, one example is [26].

Depending on their states, drivers will react differently under different conditions, leading to varied reaction time. Regarding our model, three types of drivers are presented; they are the rested drivers, tired drivers, and very tired drivers, respectively. It is not hard to imagine that rested drivers are more alert and are the fastest ones to react among all the drivers, tired drivers come second in reaction time, while very tired drivers are the slowest ones to respond.

For simplicity, in our approach, we use time steps of 10 100 for drivers' reaction time. As an example, we find time steps of 10 for rested drivers, 50 for tired ones, and 100 for the very tired ones work well in our simulation.

4.3. Integrated Model. In practice, a driver will predict according to the accumulative effects when considering certain grid points in front. The nearest grid point plays a more important role in this drivers' decision making, while the farthest one has little impact on the drivers' decision. Accordingly, we bring in a weighting function which is inversely proportional to the observed distance. To simplify this, we build a function which has an inverse relation with the distance of grid points. The function is as follows:

$$
w_{i}=\frac{b}{i-a}, \quad i \leq\left\lfloor\frac{d_{\mathrm{obs}}}{\delta}\right\rfloor,
$$


where $i$ denotes the $i$ th grid point, the first grid point is the nearest one ahead from the current position; $w_{i}$ is a weighting function, which is a monotonic decreasing function of $i$. $a$ and $b$ are certain appropriate constants, $a+b=1,0<a<1 / 2$, $b>0$, therefore the closer the grid point is, the greater the weight becomes, which means a closer grid point plays a more important role in drivers' prediction. $d_{\mathrm{obs}}$ is the observation distance.

Incorporating everything mentioned above into our method, we obtain the following integrated model:

$$
y(t+\tau)=y(t)+k \sum_{i=1}^{\left\lfloor d_{\mathrm{obs}} / \delta\right\rfloor} w_{i} \frac{\partial \mathbf{Q}(x+i \delta)}{\partial x} .
$$

In accordance with the description above, $\tau$ is drivers' reaction time and $k$ is drivers' sensitivity coefficient. $\mathbf{Q}(x+$ $i \delta)_{x}=\partial \mathbf{Q}(x+i \delta) / \partial x$ is the prediction item, which represents the drivers' effectiveness to variation of flux at grid point $i$. Equation (9) suggests that at time $t$, a driver can clearly observe some fluxes at grid points ahead based on the observation distance from his current location and then makes some prediction, after a reaction time of $\tau$ seconds, this driver may do some action as the result of his or her prediction.

We deal with (9) in the same as we did with (6), using spatial discretization, and we get a similar form as follows:

$$
y(t+\tau)=y(t)+k \sum_{i=1}^{\left\lfloor d_{\mathrm{obs}} / \delta\right\rfloor} w_{i} \frac{\mathbf{Q}(x+i \delta)-\mathbf{Q}(x)}{i \delta} .
$$

To sum up, we build up an integrated model named " ${ }^{2} \mathrm{R}$ " based on AR model, together with drivers' prediction, driver types, and drivers' reaction time. We believe that such measures can generate some natural behavior close to that of traffic in real world.

\section{Results and Discussion}

We have developed a simulation system (with OpenGL) and run a series of experiments involving 4 lanes (Figure 2). All the experiments were performed on an Intel Core(TM) i52500 3.30 GHZ CPU with 8 GB memory and an independent graphics card.

We conducted several groups of experiments in accordance with our $\mathrm{A}^{2} \mathrm{R}$ model, so as to validate our approach.

5.1. Differing Driver Types. The results of the experiments are represented in the following Figures 3, 4, and 5. In Figure 3, we report several screenshots of traffic flow from the simulation: the leftmost lane represents the AR model without considering drivers' effectiveness; from the right side to the left side, drivers in the first lane are normal, those in the second lane are aggressive, and the third lane accommodates conservative drivers. All the four lanes have the same initial conditions. We also assume that drivers all have a nice rest.

Next to the presentation of vehicle flow, we make a comparison in line with experimental results in Figure 4. The term "distance" in Figure 4 means how long a car travels,

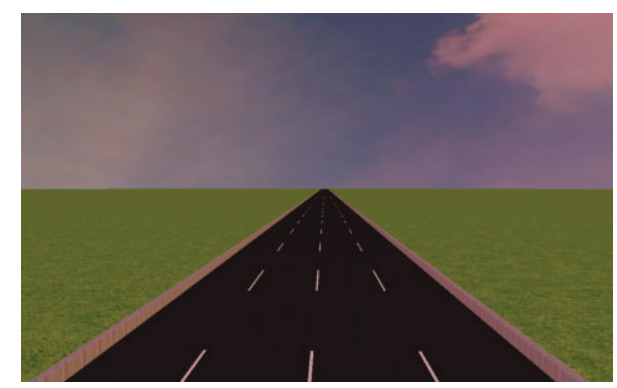

FIGURE 2: Benchmarking environment.

"interval" stands for the distance between two nearest cars in the same lane. Figure 5 gives an illustration between total simulation time and sensitivity coefficients.

Analysis. In accordance with the Figure 4, "distance" for each type of drivers will increase dramatically along with time, but "interval" usually decreases at first, and then it grows gradually for all models. Aggressive drivers are more active in velocity than others. For these three types of drivers, there is always the fact that within the same period, aggressive drivers move a longer distance than others, followed by normal and conservative ones, respectively, since aggressive ones are more sensitive to acceleration. From Figure 5, we can observe that the total time is reduced remarkably when the sensitivity coefficient $k_{+}$grows greater, which validates the effectiveness of drivers' prediction.

5.2. Varying Reaction Time. Supposing that all drivers are normal, we perform a group of experiments with diverse drivers' status.

Analysis. A driver, whose state is rested, has the shortest response time, and reacts the fastest. In line with this fact and Figure 6, the rested drivers have the largest velocity at the same time among all drivers and travel the longest distance within a certain period. By contrast, drivers who have a good rest, generally possess the smallest interval among all drivers after some time. Just as analyzed above, "interval" usually initially drops to a smallest interval and raises gradually until it reaches a steady value.

5.3. Performance. In this section, we compare the performance of agent-based model, AR model, and our $\mathrm{A}^{2} \mathrm{R}$ model. Figure 7 shows the performance of these three models on a synthetic road with 4 individual lanes. Our $\mathrm{A}^{2} \mathrm{R}$ model outperforms agent-based model by a factor of 2.5-3.5. The performance of our $A^{2} R$ model is almost the same as $A R$ model.

We also compare average velocity, number of cars, and fluxes of $\mathrm{AR}$ model and our $\mathrm{A}^{2} \mathrm{R}$ model in Figure 8. It illustrates that our model is very similar to AR model, because we keep the essence of AR model, but there is also some differences, since we introduce drivers' prediction and reaction time. 


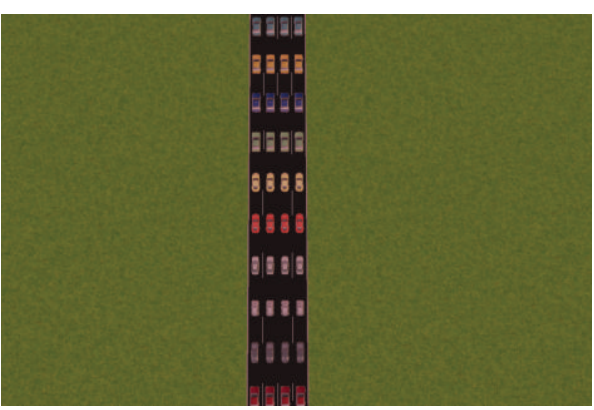

(a) $t=0 \mathrm{~s}$

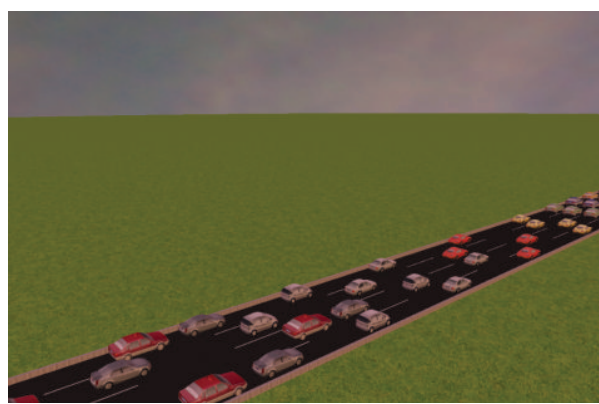

(c) $t=20 \mathrm{~s}$

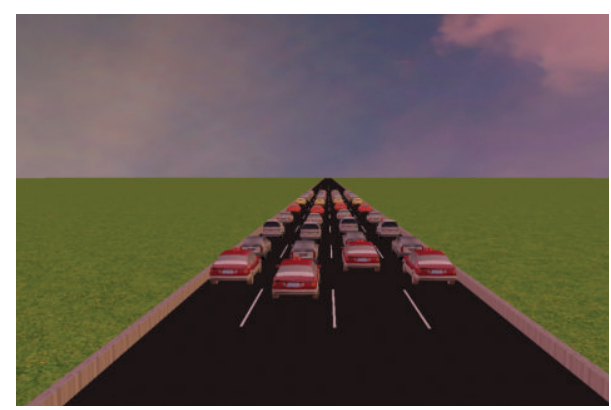

(b) $t=10 \mathrm{~s}$

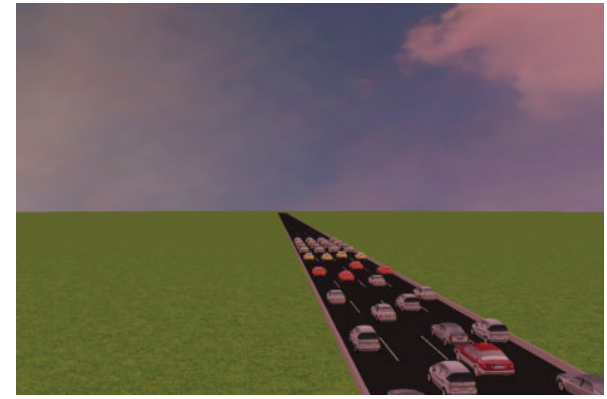

(d) $t=30 \mathrm{~s}$

FIGURE 3: Traffic flow for normal drivers, aggressive drivers, conservative drivers, and AR model.

5.4. Discussion of the Experimental Outcomes. From the results of our experiments, we can make the following observations.

(i) After a certain amount of time, drivers who are aggressive moved a longer distance, followed by normal ones, AR model, and conservative drivers, respectively. But the measure of the interval gives the opposite result. A larger sensitivity coefficient to acceleration will lead to a shorter simulation time.

(ii) After introducing the effectiveness of drivers' prediction, different states can lead to varying length of reaction time. Within the same period, drivers who are rested and alert act the fastest and move the longest distance, and the following are the tired drivers with the very tired ones taking the final place. Conversely, after some time, interval of rested drivers is the shortest, and the very tired ones have the longest interval.

The experiments we have performed show a few more promising simulation results than AR model with a similar performance.

\section{Conclusion and Future Work}

This paper has presented a novel method in traffic simulation based on AR model. Compared to AR model, our model introduces the effectiveness of drivers' prediction and reaction time, which not only reflects drivers' subjectivity but also activates vehicle flows. Combining all the considered factors with $\mathrm{AR}$ model, a new model named $\mathrm{A}^{2} \mathrm{R}$ has been built
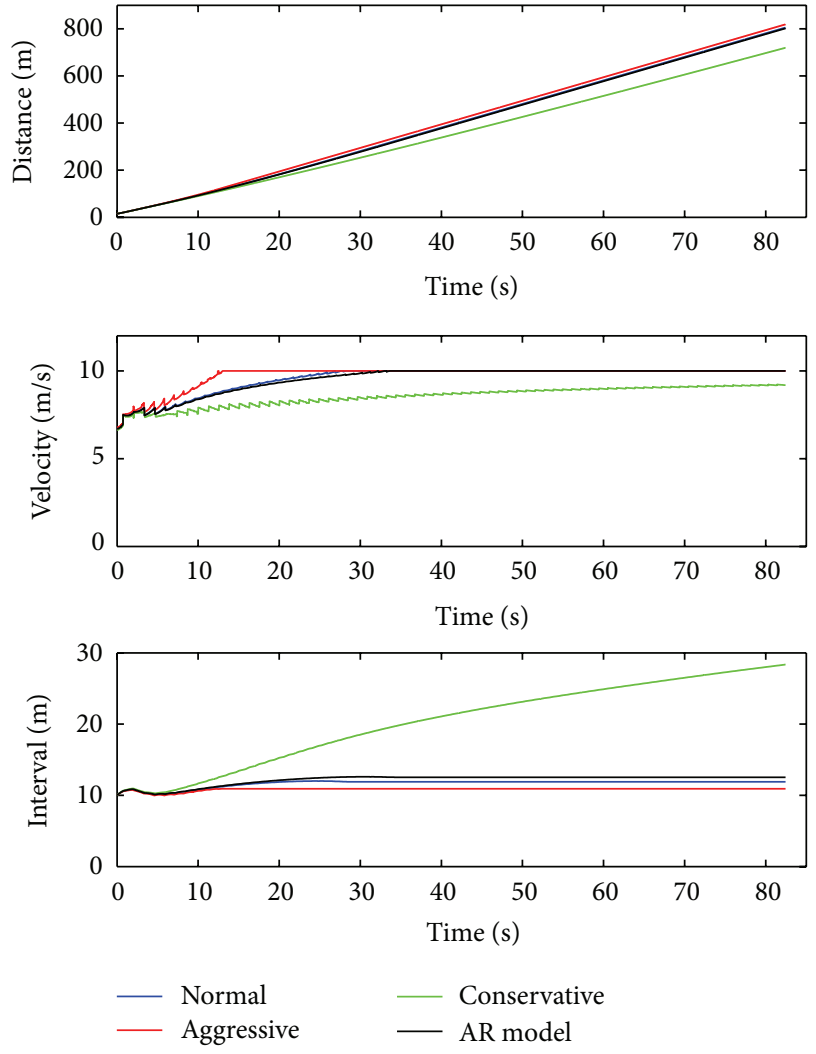

FIGURE 4: Comparison between normal drivers, aggressive drivers, conservative drivers, and AR model without considering drivers' prediction. These graphs show distance, velocity, and interval in simulation. 


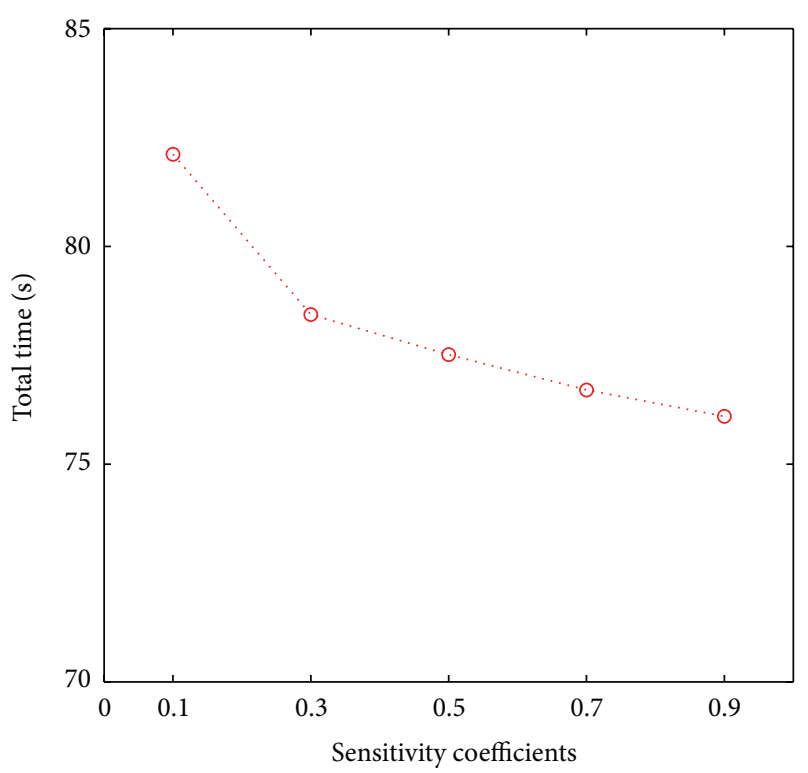

FIgURE 5: Total time of a simulation with different sensitivity coefficients $\left(k_{+}\right)$.
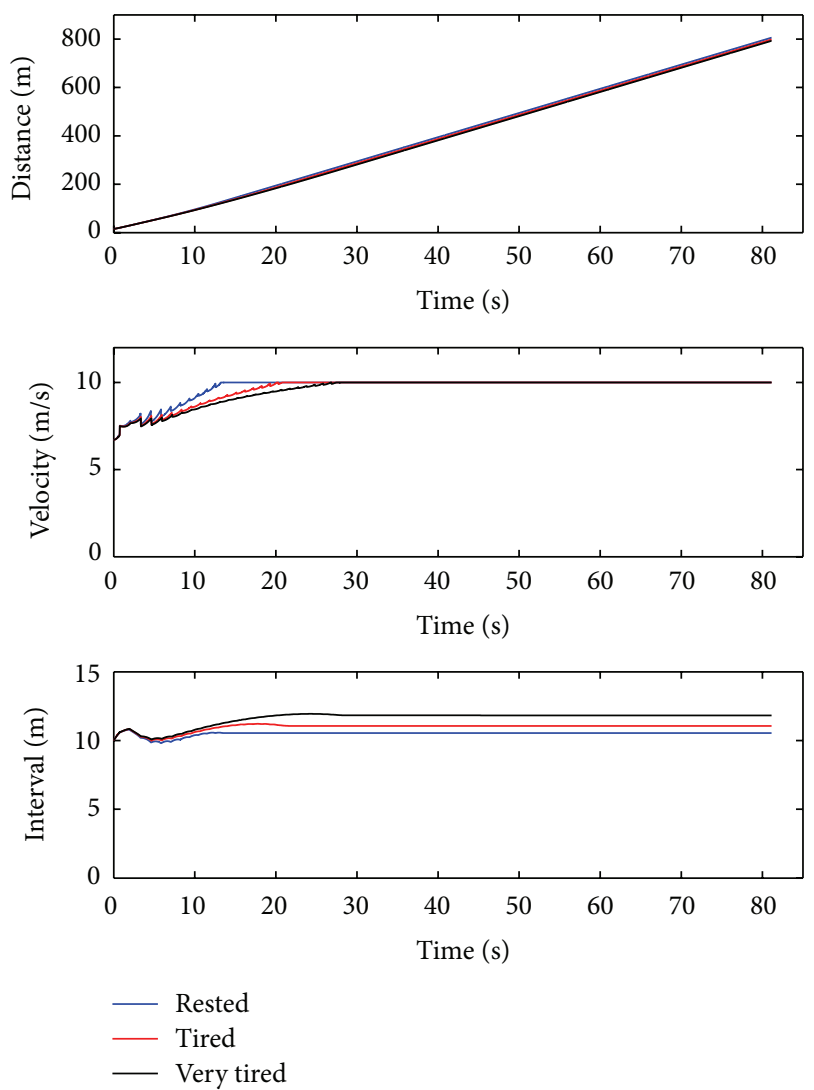

FIGURE 6: Comparison between rested drivers, tired drivers, and very tired drivers when considering varying reaction time. These three figures illustrate information of distance, velocity and interval in simulation.

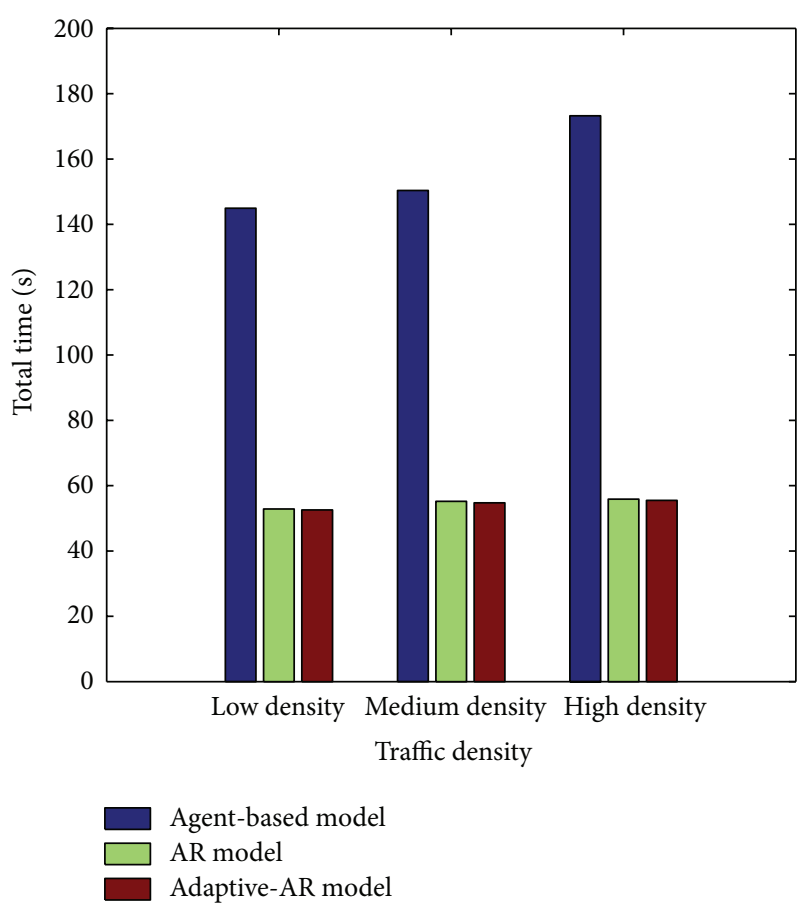

Figure 7: Performance of agent-based simulation, AR model, and our $\mathrm{A}^{2} \mathrm{R}$ model for various densities on a road network with 4 lanes.
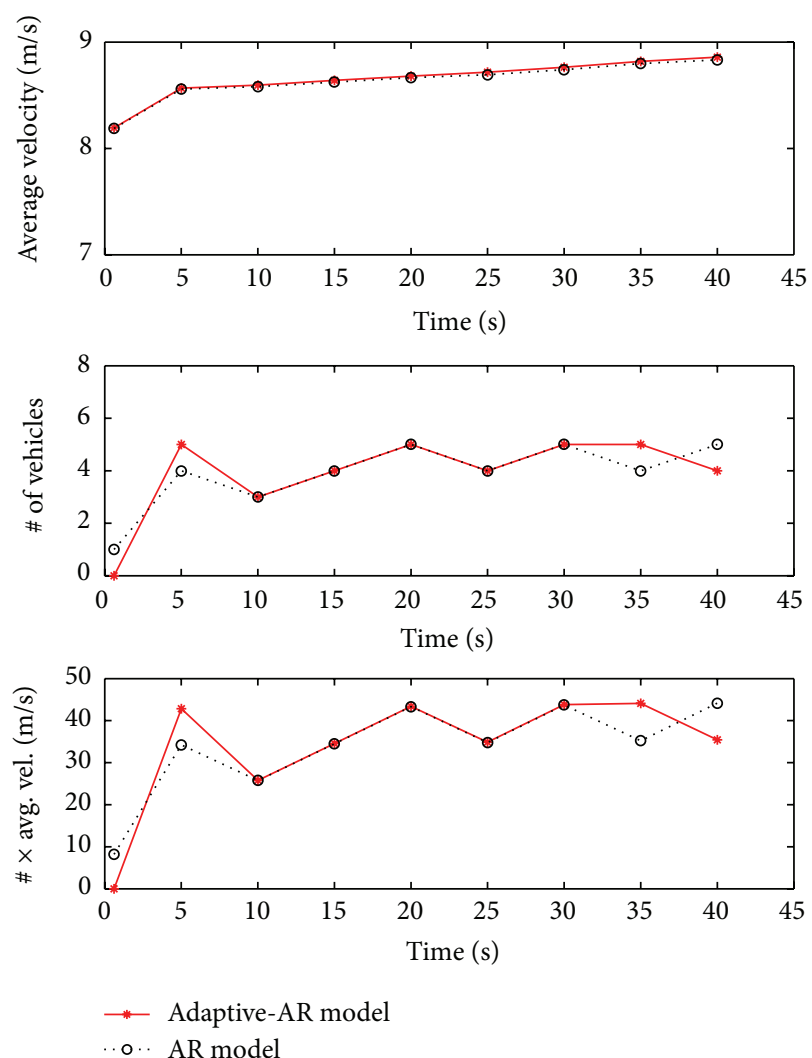

FIgUre 8: Contrast between AR model and our $\mathrm{A}^{2} \mathrm{R}$ model. These charts show velocity, density, and flux recorded over 5-second intervals. 
up. Just like other methods based on macroscopic models, our model has a much higher performance than agent-based methods. Furthermore, the consideration of many real-life factors makes vehicle flows "alive" and much closer to realworld traffic than AR model. The results in our experiments have shown that this approach is effective and lifesome in simulating vehicle flows.

Still, there are some limitations existing in the current work. What we discussed in this paper is the impact that drivers' prediction induces, which involves a positive stimulation. However, some drivers might respond to negative stimulation. For example, when the flux at a grid point ahead is greater than that at current position, a driver might decelerate in order to lower the risk of collision. Hence, in our future work, we will take negative stimulation into consideration and allow different types of drivers to be distributed evenly in each lane instead of no variation in drivers' types in a lane. Furthermore, we can simulate some specific scenarios, such as high density of traffic, to analyze the influence of drivers. On another aspect, to be more applicable, we may also turn the current work into a vehicular game and receive feedback from the users.

\section{Acknowledgments}

This research is supported by the National Science and Technology Support Program (Grant 2013BAH23F01), the Natural Science Foundation of China (Grant 60970125, 61202207, and 61210005), the China Postdoctoral Science Foundation (Grant 2012M520067 and 2013T60706), the Research Fund for the Doctoral Program of Higher Education of China (Grant 20124101120005), and the Open Project Program of the State Key Lab of CAD\&CG at Zhejiang University (Grant A1209).

\section{References}

[1] J. Sewall, D. Wilkie, P. Merrell, and M. C. Lin, "Continuum traffic simulation," Computer Graphics Forum, vol. 29, no. 2, pp. 439-448, 2010.

[2] A. Aw and M. Rascle, "Resurrection of "second order" models of traffic flow," SIAM Journal on Applied Mathematics, vol. 60, no. 3, pp. 916-938, 2000.

[3] D. L. Gerlough, Simulation of Freeway Traffic on a GeneralPurpose Discrete Variable Computer, University of California, Los Angeles, Calif, USA, 1955.

[4] G. Newell, "Nonlinear effects in the dynamics of car following," Operations Research, vol. 9, no. 2, pp. 209-229, 1961.

[5] S. Algers, E. Bernauer, M. Boero et al., "Smartest project: review of micro-simulation models," EU Project RO-97-SC, 1059, 2, 1997.

[6] D. Helbing, "Traffic and related self-driven many-particle systems," Reviews of Modern Physics, vol. 73, no. 4, pp. 1067-1141, 2001.

[7] K. Nagel and M. Schreckenberg, "A cellular automation model for freeway traffic," Journal de Physique I, vol. 2, no. 12, pp. 22212229, 1992.

[8] M. Treiber, A. Hennecke, and D. Helbing, "Congested traffic states in empirical observations and microscopic simulations," Physical Review E, vol. 62, no. 2, pp. 1805-1824, 2000.
[9] MITSIM, MIT Intelligent Transportation Systems, 2012.

[10] SUMO-Simulation of Urban Mobility, 2012, http://sumo-sim .org/.

[11] M. J. Lighthill and G. B. Whitham, "On kinematic waves. II. A theory of traffic flow on long crowded roads," Proceedings of the Royal Society of London A, vol. 229, no. 1178, pp. 317-345, 1955.

[12] P. I. Richards, "Shock-waves on the highway," Operations Research, vol. 4, no. 1, pp. 42-51, 1956.

[13] H. J. Payne, "Models of freeway traffic and control," in Mathematical Models of Public Systems I, pp. 51-60, Society for Computer Simulation, San Diego, Calif, USA, 1971.

[14] H. M. Zhang, "A non-equilibrium traffic model devoid of gaslike behavior," Transportation Research B, vol. 36, no. 3, pp. 275290, 2002.

[15] I. Prigogine and F. C. Andrews, "A Boltzmann-like approach for traffic flow," Operations Research, vol. 8, no. 6, pp. 789-797, 1960.

[16] P. Nelson, D. Bui, and A. Sopasakis, "A novel traffic stream model deriving from a bimodal kinetic equilibrium," in Proceedings of the International Federation of Automatic Control Meeting (IFAC '97), Chania, Greece, 1997.

[17] V. Shvetsov and D. Helbing, "Macroscopic dynamics of multilane traffic," Physical Review E, vol. 59, no. 6, pp. 6328-6339, 1999.

[18] T. Nagatani, "Modified KdV equation for jamming transition in the continuum models of traffic," Physica A, vol. 261, no. 3-4, pp. 599-607, 1998.

[19] D.-H. Sun and C. Tian, "A traffic flow lattice model with the consideration of driver anticipation effect and its numerical simulation," Acta Physica Sinica, vol. 60, no. 6, Article ID 068901, 2011.

[20] J. Go, T. D. Vu, and J. J. Kuffner, "Autonomous behaviors for interactive vehicle animations," Graphical Models, vol. 68, no. 2, pp. 90-112, 2006.

[21] J. Sewall, J. van den Berg, M. C. Lin, and D. Manocha, "Virtualized traffic: reconstructing traffic flows from discrete spatiotemporal data," IEEE Transactions on Visualization and Computer Graphics, vol. 17, no. 1, pp. 26-37, 2011.

[22] D. Wilkie, J. van den Berg, M. Lin, and D. Manocha, "Selfaware traffic route planning," in Proceedings of the 25th AAAI Conference on Artificial Intelligence (AAAI '11), pp. 1521-1527, San Francisco, Calif, USA, August 2011.

[23] D. Wilkie, J. Sewall, and M. C. Lin, "Transforming GIS data into functional road models for large-scale traffic simulation," IEEE Transactions on Visualization and Computer Graphics, vol. 18, no. 6, pp. 890-901, 2012.

[24] X. Lu, W. Chen, M. Xu, Z. Wang, Z. Deng, and Y. Ye, "AAFVDM: an accident-avoidance full velocity difference model for animating realistic street-level traffic in rural scenes," Computer Animation and Virtual Worlds, 2013.

[25] J. Sewall, D. Wilkie, and M. C. Lin, "Interactive hybrid simulation of large-scale traffic," ACM Transactions on Graphics, vol. 30, no. 6, article 135, 2011.

[26] M. T. Corfitsen, "Tiredness and visual reaction time among young male nighttime drivers: a roadside survey," Accident Analysis and Prevention, vol. 26, no. 5, pp. 617-624, 1994.

[27] R. Courant, K. Friedrichs, and H. Lewy, "On the partial difference equations of mathematical physics," IBM Journal of Research and Development, vol. 11, no. 2, pp. 215-234, 1967.

[28] Y. Liu, G.-L. Chang, and J. Yu, "Empirical study of driver responses during the yellow signal phase at six maryland intersections," Journal of Transportation Engineering, vol. 138, no. 1, pp. 31-42, 2012. 

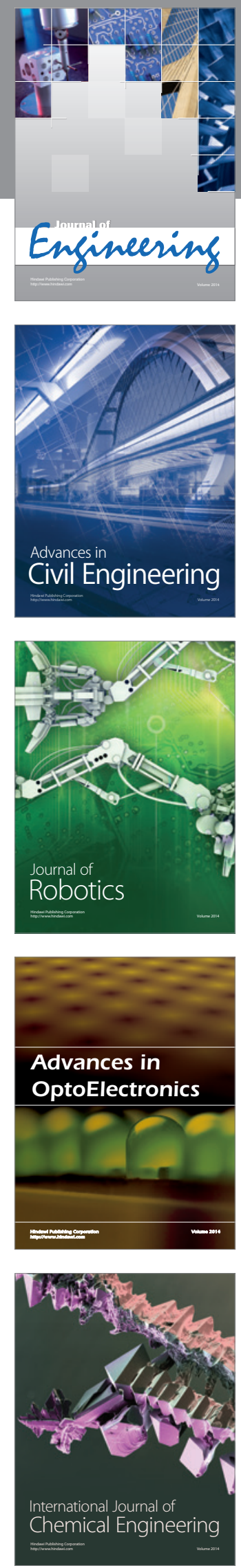

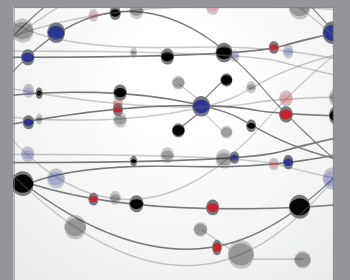

The Scientific World Journal
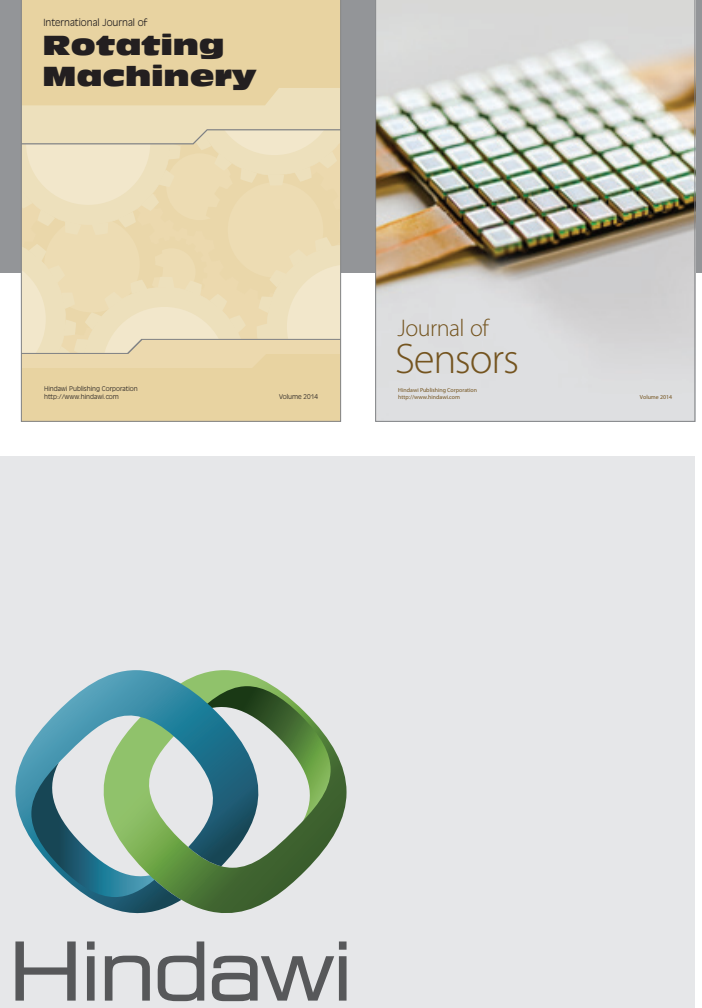

Submit your manuscripts at http://www.hindawi.com
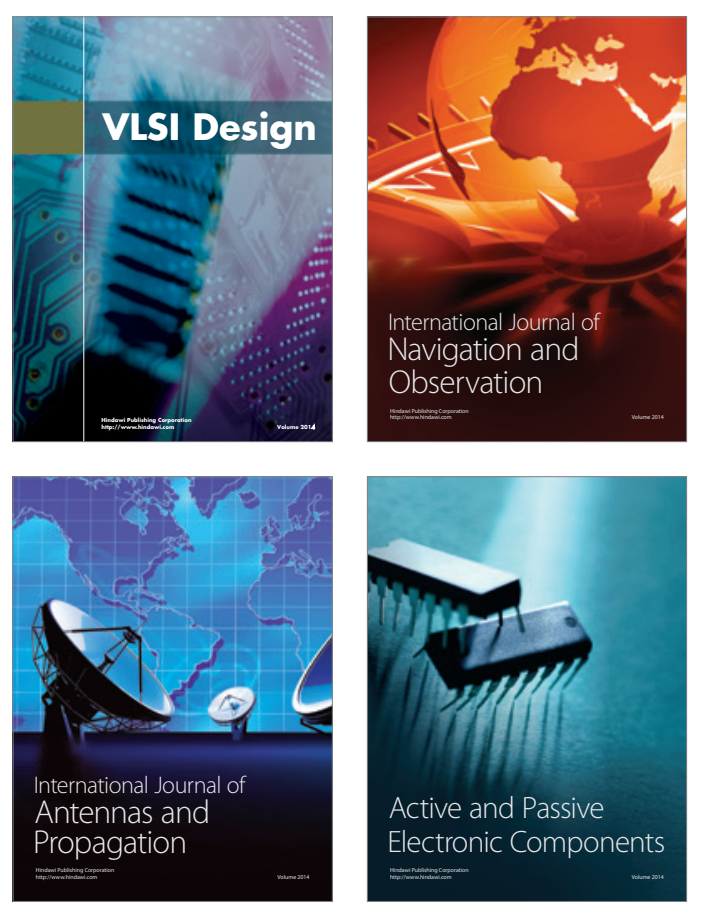
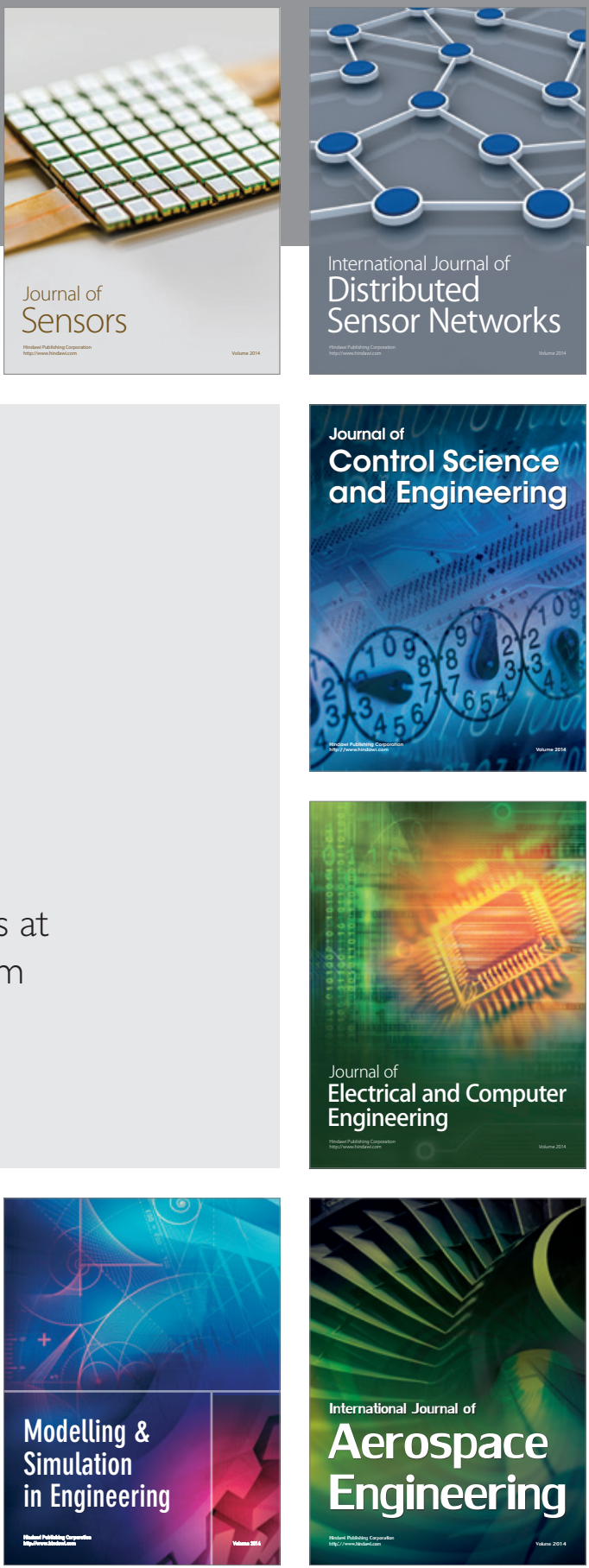

Journal of

Control Science

and Engineering
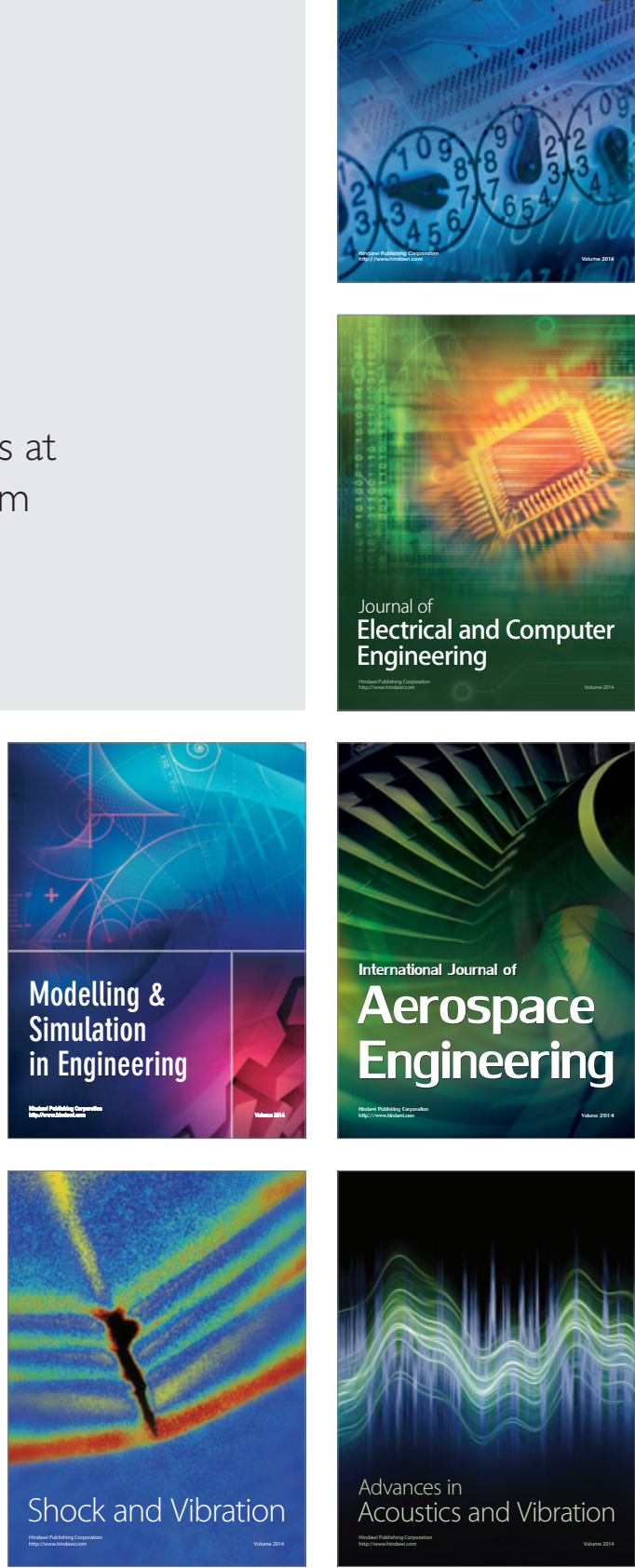